\title{
Applications of 3D Body Scanning Technology to Human Anthropometry: Body Surface Area and Body Volume Measurements in the Fields of Health and Sports Sciences
}

\author{
Kazuo FUNATO ${ }^{a}$, Noriko HAKAMADA ${ }^{a}$, Hidehiko NAGASHIMA ${ }^{\mathrm{b}}$, Chiyoharu HORIGUCHI ${ }^{\mathrm{b}}$ \\ ${ }^{a}$ Laboratory for Human Movement Sciences, Nippon Sport Science University, Yokohama, Japan; \\ ${ }^{\mathrm{b}}$ Hamamatsu Photonics K.K., Hamamatsu, Japan
}

\begin{abstract}
In the fields of sports and health sciences, changes in body shape are one of the most important parameters in order to evaluate the effects of physical training and /or routine workout performed. Until now, these parameters were typically measured under manually skilled techniques in anthropometry. In recent years, Whole body measurement system for humans (that is called "Bodyline Scanner", Hamamatsu Photonics K.K., Japan) which has functions such as a high speed measurement and high resolutions are required by many industrial and academic areas such as apparel and digital technology. The purposes of present study were to develop 3D anthropometry by optical triangle method using laser beam system and to determine feasibility of the measurements such as lengths, circumferences, body surface area (BSA) and body/segment volumes with comparing to other conventional methods. Newly developed Bodyline scanner (BLS) as shown in Fig.1 was capable of digitizing whole body shape as three dimensional coordinates in the order of $2.5 \mathrm{~mm}$ intervals in space (normal adult body shape put in $\sim 500,000$ points). The principle of the measuring method was optical triangle measurement, in which light source was using a laser diode. The color information was used to detect the position of land mark seals which was pasted on the skin according to the anatomical basis in human anthropometry. Six moving scanner heads and intelligent vision camera systems were enable to reduce the area which can not measure those positions such armpit and crotch, and to reduce whole body scanning time up to ten seconds. Measuring height was as much as $2300 \mathrm{~mm}$ which enable to scan whole body from children to such one as Olympic basketball athletes. 3D polygon data reconstructed after digitizing each point was used for the measurement of anthropometry such as lengths and circumferences of each limb or trunk, body surface area (BSA) and each segment volumes or whole body volume. Sixty seven males and females aged from 9 to $21 y$ rs were tested in both BLS and manually conventional tape measure methods (TAPE).

Differences of lengths and circumferences of the limbs between BLS and TAPE were within 4\%. BSA data was almost identical to those estimated from the equation based on body height and weight ( $\mathrm{Du}$ Bois, 1916). Whole body volume data obtained from BLS were precisely consistent with those obtained from air displacement methods.

Anthropometric data as well as body surface area and body volume measured by using 3D body scanning technologies might be widely prospective for evaluating the differences or changes in body shape in such fields as health and sports sciences.
\end{abstract}

Key words: 3D Anthropometry, Body surface area, Body volume, Health and sports sciences

\section{Introduction}

Segmental anthropometry, which measures segment masses, segment lengths, segment center-of-mass locations, and segment moments of inertia can be determined by a number of different means. They have been derived from cadavers [1, 2], from direct measument [3], from regression equations, and from mathematical modelling [4]. The choice of body segment parameters determined from such means might be based on the population being studied and level of accuracy sought by the researcher. Recently, another approach to estimating body segment parameters involves scanning the living body with various techniques such as gamma mass scanning, photogrammetry, MRI and DEXA. Whole Body Measurement System for humans "Bodyline Scanner" which has functions such as a high speed measurement and high resolutions are required by many industrial and academic areas such as apparel and digital technology.

*Email: funato@nittai.ac.jp, web: http://www.nittai.ac.jp 
The purposes of the present study were to develop 3D anthropometry by optical triangle method using laser beam system and to determine feasibility of segment parameter measurements such as lengths, circumferences, body surface and body/segment masses with comparing to previous studies.

\section{Methods}

As shown in Fig.1, newly developed Bodyline scanner (BLS) was capable of digitizing whole body shape as three dimensional coordinates in the order of $2.5 \mathrm{~mm}$ intervals in space (normal adult body shape put in $\sim 500,000$ points). The principle of the measuring method was optical triangle measurement, in which light source was using a laser diode. The color information was used to detect the position of land mark seals which was pasted on the skin according to the anatomical basis in human anthropometry. Six moving scanner heads and intelligent vision camera systems were enable to reduce the area which can not measure those positions such armpit and crotch, and to reduce whole body scanning time up to 5 seconds.

Features of BLS newly developed were as follows;

- Optical Cross Sectioning using Slit LASER and special CCD camera

- Triangulation Method

- Visible Slit LASER with Eye-safe Class 1

- High Resolution (1mm) and Accuracy (+/- 0.5\%)

- High Scan Speed at 5 ( $5 \mathrm{~mm}$ pitch) or $10 \mathrm{sec}(2.5 \mathrm{~mm}$ pitch)

- 4 Sensor Modules with Simple Structure for Easy Set-up and Maintenance

- Combine with Bodyline Manager

More detailed features of BLS were indicated in Appendix A-E.

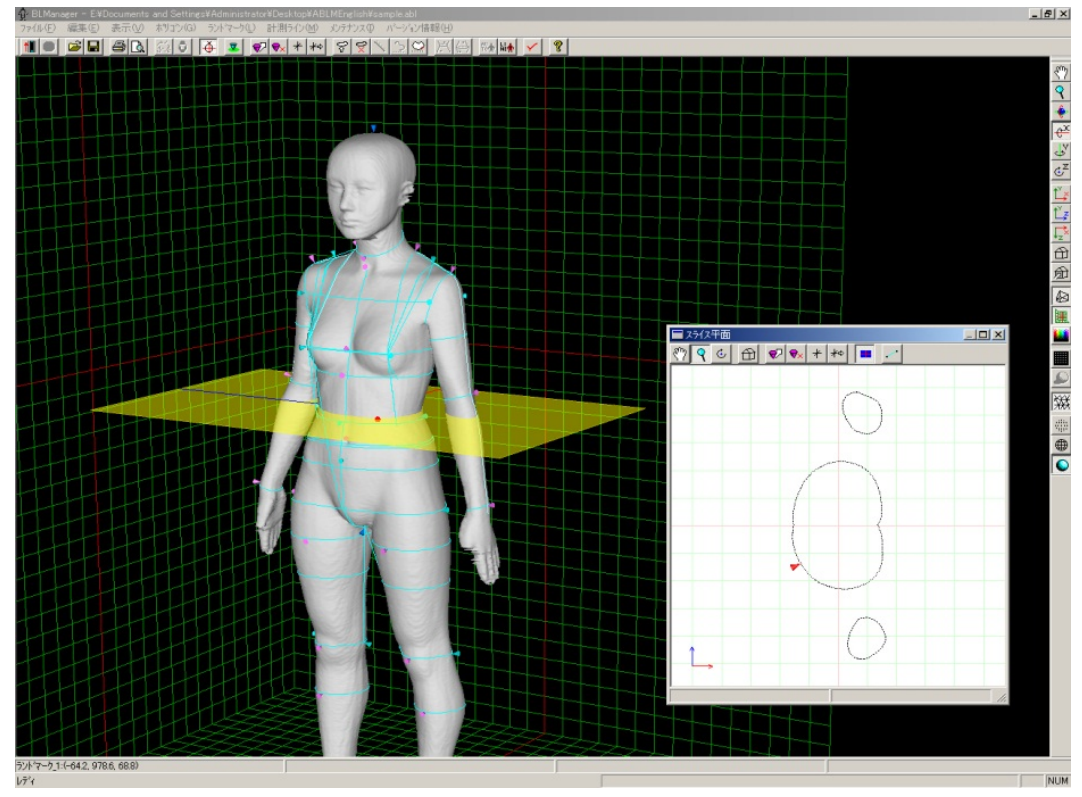

Fig. 1. Laser beam sheet scanning during human standing posture for determining 3D anthropometric data. Inserted graph shows cross-section, which can get every $2.5 \mathrm{~mm}$ interval for vertical axis.

Subjects were Japanese sixty seven males and females aged from 9 to 21yrs males. (males: 23.8yrs, $171.5 \mathrm{cnBH}, 68.7 \mathrm{kgBW}$, female: $27.4 \mathrm{yrs}, 156.7 \mathrm{cmBH}, 54.4 \mathrm{kgBW}$ in averages) Each segment length and circumference were measured based on anatomical landmark points as indicated in Fig.2. For each subject, identical segment length and circumference data were taken by tape measuring conventional manual methods.

Scanning whole body data were dissected into each segment as the same manner as Clauser CE, et al. [1]. After segmental length, circumference and volume were calculated, segment masses were determined by multiplying segmental volume to respective segmental density obtained from previous study [1]. 


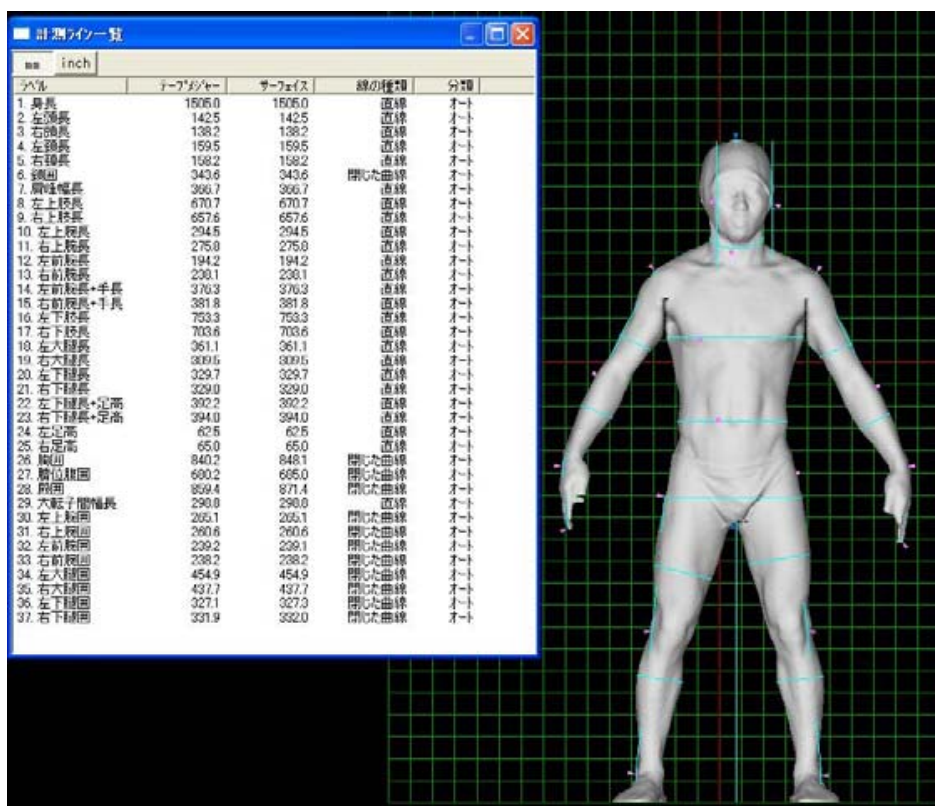

Fig. 2. Example of each segment length and circumference data automatically measure based on anatomical landmark points from BLS.

Body surface area (BSA) was calculated as sum of area of each triangle which consist the surface of reconstructed polygon data as indicated in Fig. 3. BSA obtained from BLS was compared with BSA estimated by the equation from Body height and weight indicated as Du Bois in 1916 (Fig.3.).

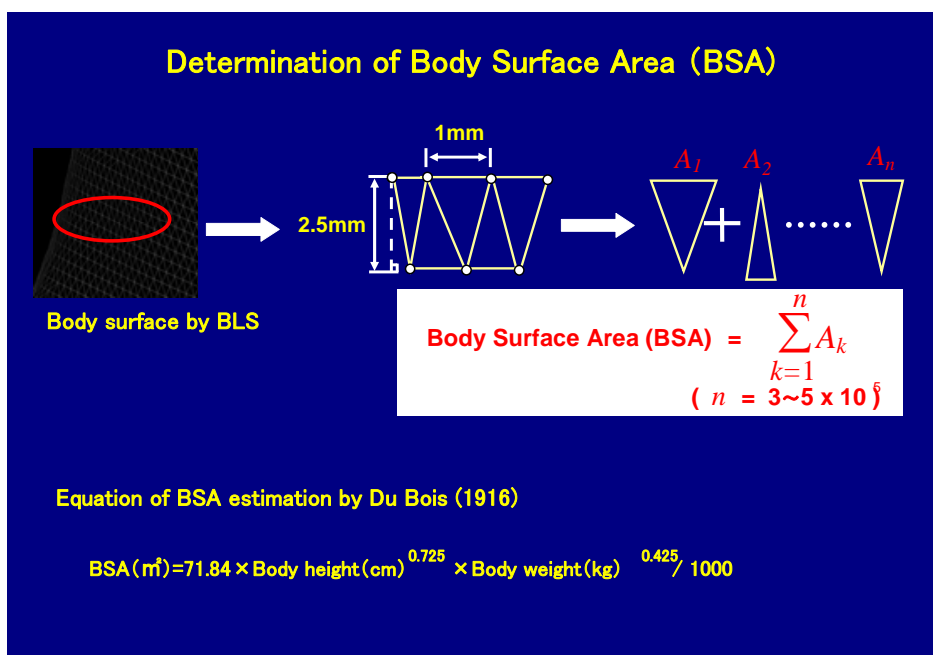

Fig. 3. Calculation of body surface area (BSA) from reconstructed polygon data.

Area of each triangle which consist the surface of whole body was summed. BSA was also estimated by the equation from Body height and weight indicated as Du Bois in 1916.

\section{Results and Discussion}

Segment limb lengths measured from BLS and directly tape measured were almost identical as shown in Fig. 4. $\left(Y=1.002 X+5.743, r^{2}=0.998, \%\right.$ Diff. $\left.=1.7\right)$. Similar relation was observed in segment kimb circumferences in Fig. 5. ( $Y=0.961 X+5.066, r^{2}=0.996, \%$ Diff. $\left.=-2.8\right)$.

Table 1 indicated \%Differences in each segment length and circumference obtained between BLS and directly tape measured. In segment length relatively higher \%Differences were observed in upper and fore arm, on the other hand, trunk region such as chest and abdominal showed relatively higher \% Differences in segment circumferences. 
Identical close correlation was observed in whole body volumes measured between BLS and directly measured by air displacement methods in Fig. 6. $\left(Y=0.987 X+1.025, r^{2}=0.997, \%\right.$ Diff. $\left.=-3.1\right)$. Comparison of whole body surface area (BSA) measured from BLS and estimated by Du Bois's equation was shown in Fig. 7. Also in whole body surface area, identical close correlation was observed between BLS directly measured and estimated value. $\left(Y=1.053 X+0.096, r^{2}=0.979, \%\right.$ Diff. $=-0.5$ ).

Table 2 showed relative segment mass to body weight (unit: \%) for each segment and comparison to previous studies. \% segment mass estimated by BLS in this study have a tendency to be smaller in trunk as well as to be larger thigh and calf in lower limb compared to the respective values previously obtained by cadavers measurements in Dempster, 1958 and Clanser, 1969. Relative segment mass to body weight in each segment was very similar to previous studies except for the segments of trunk and thigh (Table 2). Previous relative segment mass values have a tendencies for overestimation in trunk, whereas underestimation in thigh. This should take into account for calculating mechanical parameters in biomechanical research.

In general, differences of lengths and circumferences of the limbs between BLS and TAPE were within $4 \%$. BSA data was identical to those estimated from body height and weight( Du Bois, 1916). The volumes of whole body and each segment are prospective for both biomechanical and biomedical fields.

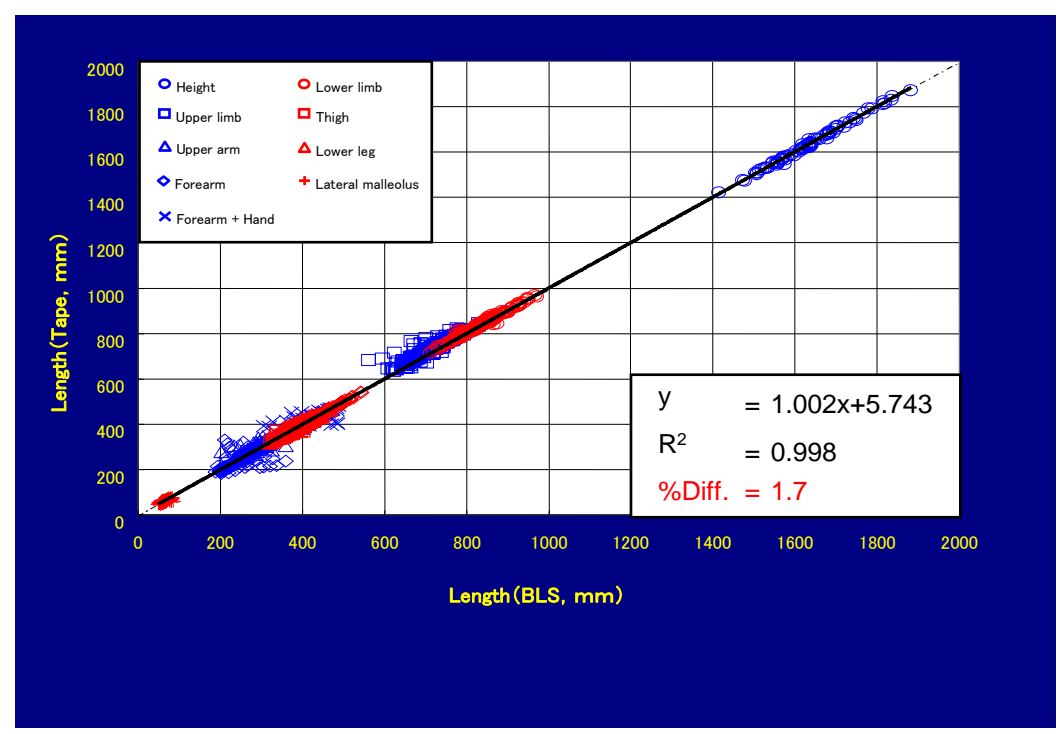

Fig. 4. Comparison of segment limb lengths measured from BLS and directly tape measured.

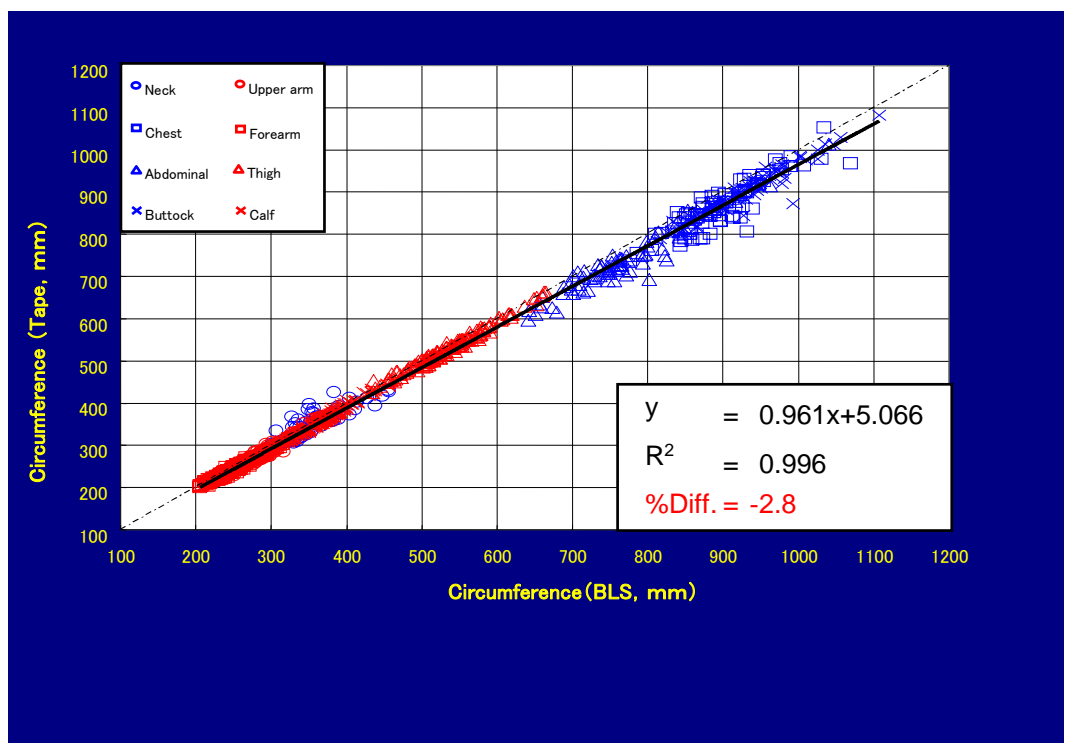

Fig. 5. Comparison of segment limb circumferences measured from BLS and directly tape measured. 
Table 1. \%Differences in each segment length and circumference obtained between BLS and directly tape measured.
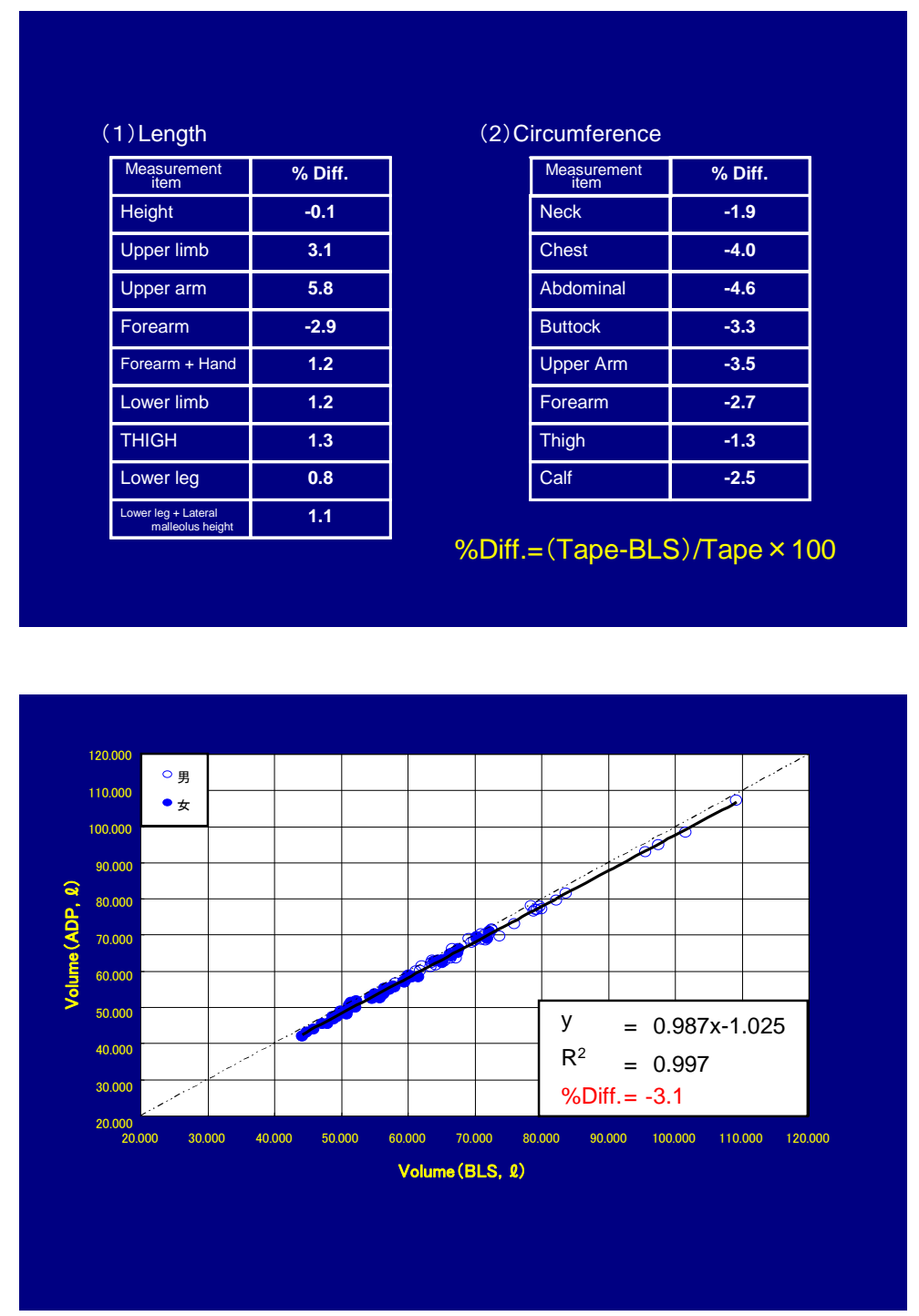

Fig. 6. Comparison of whole body volumes measured from BLS and directly measured by air displacement methods.

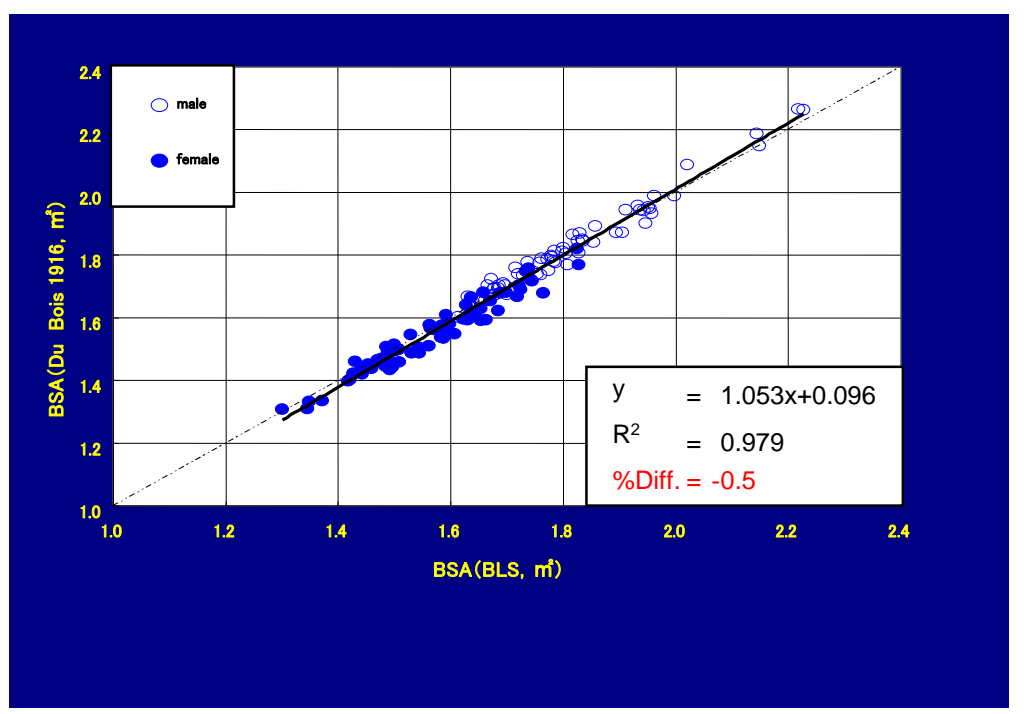

Fig. 7. Comparison of whole body surface area (BSA) measured from BLS and estimated by Du Bois's equation. 
Table 2. Relative segment mass to body weight (unit: \%) for each segment and comparison to previous studies.

\begin{tabular}{|lcccc|}
\hline & Present & Dempster & Clauser & Cheng \\
\# of subj. & 2008 & 1955 & 1969 & 2000 \\
Methods & 12 & 8 & 13 & 8 \\
\hline Head & BLS & Cadavers & Cadavers & MRI \\
Trunk & 7.3 & 8.1 & 7.3 & 7.7 \\
U-arm & 45 & 49.7 & 50.7 & 46.2 \\
F-arm & 2.7 & 2.8 & 2.6 & 4 \\
Hand & 1.7 & 2.2 & 1.6 & 1.5 \\
Thigh & 0.6 & 0.6 & 0.7 & 0.7 \\
Calf & 12.9 & 9.9 & 10.3 & 13.6 \\
Foot & 4.8 & 4.6 & 4.3 & 4.4 \\
\hline
\end{tabular}

\section{Conclusions}

1. Validity and accuracy of laser beam scanning anthropometry might be established with comparison of conventional tape measure with overall \% deference of 1.7 in length measures and 2.8 in circumference measures.

2. Body volume and body surface area calculated by BLS were closely identical to those measured by air displacement method (BOD POD) and estimation equation by Du Bois(1916), respectively.

3. In relative segmental mass to body weight, lower trunk value and higher thigh value were indicated by comparing to previous studies in cadavers.

4. Particularly whole body volumes and each segment volumes are prospective for both biomechanical and biomedical research fields.

\section{References}

1. Chen CK, et al. Clin Biomech 15: 559-566, 2000.

2. Clauser CE, et al. AMRL Technical Report :60-70, Wright-Patterson Air Force Base, OH, 1969.

3. Dempster, WT. WADC Technical Report :55-159, Wright-Patterson Air Force Base, OH, 1955.

4. Hanavan, EP. AMRL Technical Report :64-102, Wright-Patterson Air Force Base, OH, 1964.

5. Zatsiorsky, VM and Seluyanov, VN. Biomechanics IX-B (ed. Winter, DA et al.): 233-9, Human Kinetics, 1985.

\section{Appendix A}

\section{C9036 Bodyline Scanner Specification}

- \# of head : 4 consisting of special CCD camera and Visible LASER

- Light Source Visible LASER : Class 1

- Measurable Range

Height : $1,850 \mathrm{~mm}(2,000 \mathrm{~mm}$ is possible at C9036-02)

Depth : $600 \mathrm{~mm}$

Width : $1,000 \mathrm{~mm}$

Measuring Horizontal Pitch at center : $1 \mathrm{~mm}$

- Vertical pitch : $2.5 \mathrm{~mm}$ or $5 \mathrm{~mm}$ selectable

- Accuracy : $+/-0.5 \%$ (Cylinder with $1000 \mathrm{~mm}$ perimeter )

- \# of Points / one slice

Horizontal :2,560 points

Vertical : 740 or 370 points $(2.5 \mathrm{~mm}$ or $5 \mathrm{~mm}$ pitch)

Total data size : $1,894,400$ or 947,200 ( $2.5 \mathrm{~mm}$ or $5 \mathrm{~mm}$ pitch)

Scanning time : $5 / 10$ sec selectable

Dimension : 2,300mm x 1,800mm x 1,800mm (2,450mm for 2000mm) 


\section{Appendix B}

\section{Specification of C9036-02}

Type name

Measurable range

Accuracy

Measurement time

Measurement points

Power consumption

Dimensions

Weight
C9036-02

$$
\begin{array}{lc}
\text { Height } & 2000 \mathrm{~mm} \\
\text { Depth } & 600 \mathrm{~mm} \\
\text { Width } & 1000 \mathrm{~mm} \\
\pm 0.5 \%(1000 \mathrm{~mm} \text { perimeter as standard) } \\
5.4 \mathrm{sec} / 10.8 \mathrm{sec} \\
\text { 1,024,000/2,048,000points } \\
700 \text { VA } \\
\text { Height } & 2450 \mathrm{~mm} \\
\text { Depth } & 1800 \mathrm{~mm} \\
\text { Width } & 1800 \mathrm{~mm}
\end{array}
$$

\section{Appendix C}

\section{Work Flow of BLM}

- Take the image data from BLS

- Translate from image data to distance data

- Reject noise data and translate to $X, Y, Z$ coordinates

- Complement, Filling and smoothing

- Detect basic 5 Landmarks

- Segmentation of a body

- Reconstruct polygon data

- Detect the Landmark

- Auto-measurement

\section{Appendix D}

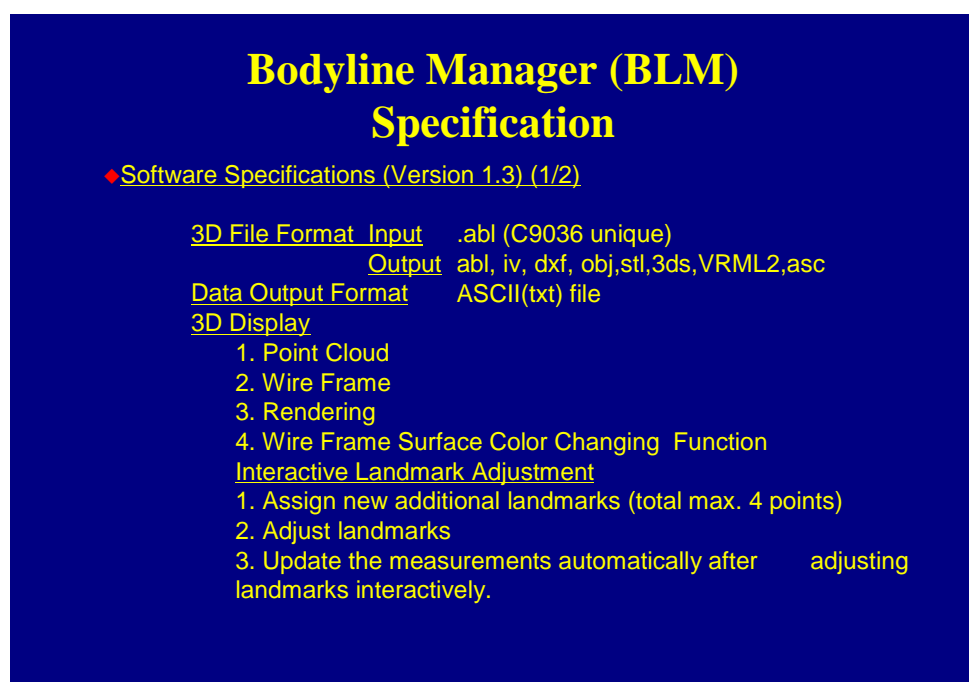




\section{Appendix E}

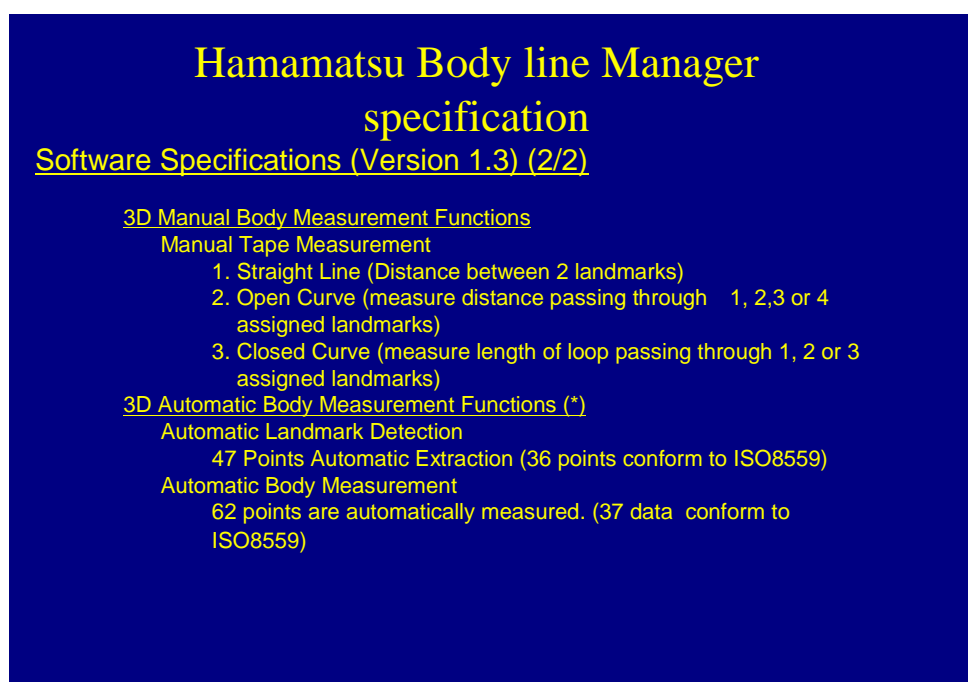

